Original research article

\title{
Pre-treatment with Empagliflozin ameliorates Cisplatin induced acute kidney injury by suppressing apoptosis
}

\author{
Maaly A. Abd Elmaaboud ${ }^{1}{ }^{*}$, Ahmed M. Kabel ${ }^{1,2}$, Mohamed Elrashidy ${ }^{3}$ \\ ${ }^{1}$ Tanta University, Faculty of Medicine, Department of Pharmacology, Tanta, Egypt \\ ${ }^{2}$ Taif University, College of Pharmacy, Department of Clinical Pharmacy, Taif, Saudi Arabia \\ ${ }^{3}$ Tanta University, Faculty of Medicine, Department of Pathology, Tanta, Egypt
}

\section{Abstract}

Dose-limiting nephrotoxicity restricts Cisplatin use in high therapeutic doses. Empagliflozin showed a reno-protective effect in diabetic nephropathy. We investigated if Empagliflozin can ameliorate Cisplatin nephrotoxicity whether used prophylactically or therapeutically. Forty male Wistar rats were divided into 5 groups: (1) control; (2) Cisplatin-induced nephrotoxicity by single intraperitoneal dose; (3) Empagliflozin was given for 10 days before a single dose of Cisplatin; (4) a single dose of Cisplatin followed by Empagliflozin for 10 days; (5) received Empagliflozin only. Regular assessment of weight was done, biochemical evaluation for serum urea, creatinine, uric acid, albumin, and glucose was performed, kidney tissue nerve growth factor- $\beta$ (NGF- $\beta$ ) and oxidative stress parameters were measured, kidneys were evaluated histopathologically and immunostained for caspase 3. Cisplatin significantly reduced body weight, NGF- $\beta$, and reduced glutathione, elevated urea, creatinine, and malondialdehyde with no effect on other serum biochemical parameters. Histopathologically, there was high acute tubular necrosis (ATN) score with strong immunostaining of caspase 3. The use of Empagliflozin significantly reduced urea and creatinine in both prophylactic and therapeutic, reduced ATN score in the prophylactic group associated with minimal staining of caspase 3 and elevated reduced glutathione. In conclusion, prophylactic Empagliflozin protected against Cisplatin-induced acute kidney injury mainly via anti-apoptotic effect.
\end{abstract}

Keywords: Apoptosis; Cisplatin; Empagliflozin; Nerve growth factor; Reduced glutathione

Highlights:

- Empagliflozin has a direct nephroprotection effect independent on glycemic control.

- Empagliflozin protects kidney from tubular damage caused by Cisplatin via suppressing apoptosis.

- Administration of Empagliflozin after injury confers no reversing effect.

- Nerve growth factor- $\beta$ has a relation to Cisplatin toxicity.

\section{Introduction}

Cisplatin is one of the most valuable chemotherapeutic agents which is used in a wide variety of malignancies including head, neck, ovarian, testicular, lung cancer and refractory non-Hodgkin lymphoma. Its effectiveness in cancer arises from interactions with DNA, induction of oxidative stress and apoptosis (Dasari and Tchounwou, 2014). Despite its effectiveness, its use is limited by tumor cell resistance and adverse effects such as neurotoxicity, ototoxicity, and nephrotoxicity (Barabas et al., 2008; Sancho-Martínez et al., 2012). Nephrotoxicity in the form of acute kidney injury is the most common and serious adverse effect. It occurs even after a single dose in one-third of patients which limits increasing the dose hence impairing treatment effectiveness (Miller et al., 2010).

It is known that Cisplatin induces its injurious effect on kidney mainly on S3 portion of proximal convoluted tubules in form of acute tubular necrosis. Several different mechanisms have been proposed: accumulation and activation into toxic metabolite, DNA damage, enhanced oxidative damage, mitochondrial dysfunction, inflammation, intracellular messengers and transducers and induction of apoptosis and necrosis (Peres and da Cunha, 2013).

Many strategies were tried in patients to prevent nephrotoxicity; as hydration, diuretics and magnesium administration aiming to decrease Cisplatin concentration in renal cells and enhancing its excretion. Recently, it was accepted that short hydration and forced diuresis with mannitol, not frusemide may be safer in preventing Cisplatin nephrotoxicity (Crona et al., 2017). However, this evidence is still limited as mannitol is used in selected cases and may be nephrotoxic in large doses. Further research about hydration is needed to evaluate it in different ages and practice settings (Duffy et al., 2018).

Empagliflozin is an antidiabetic drug which acts as sodium glucose transporter (SGLT2) inhibitor in proximal convoluted

\footnotetext{
* Author for correspondence: Maaly Ahmed Abd Elmaaboud, Tanta University, Faculty of Medicine, Department of Pharmacology, El-Gaish, 31527 Tanta Quism 2, Egypt; e-mail: maali_abdelmaboud@med.tanta.edu.eg http://doi.org/10.32725/jab.2019.003

Submitted: 2018-08-31 • Accepted: 2019-01-23 • Prepublished online: 2019-02-05

J Appl Biomed 17/1: 82-90 • EISSN 1214-0287 • ISSN 1214-021X

(c) 2019 The Authors. Published by University of South Bohemia in České Budějovice, Faculty of Health and Social Sciences.

This is an open access article under the CC BY-NC-ND license.
} 
tubules. Its antidiabetic effect arises from inhibition of absorption of $90 \%$ of filtered glucose (Neumiller, 2014). Recently, it was found that Empagliflozin may have a reno-protective activity in diabetic nephropathy not only through its glycemic control effect but also through blood pressure lowering and direct renal effects - inhibition of inflammatory and fibrotic responses of renal proximal tubular cells to hyperglycemia (Fioretto et al., 2016; Škrtić and Cherney, 2015).

This research was conducted to investigate whether Empagliflozin has a reno-protective or therapeutic effect on Cisplatin-induced acute kidney injury irrespective of its antidiabetic and glucosuria action, especially in relation to its effect on apoptosis, oxidative stress, and inflammation.

\section{Materials and methods}

\section{Animals and ethical decision}

40 Wistar male rats aged (6-8 weeks old) weighing (120$150 \mathrm{~g}$ ) were obtained from Tanta University. They were maintained for 2 weeks under standard laboratory conditions of temperature $\left(25 \pm 3^{\circ} \mathrm{C}\right)$, relative humidity $(60 \pm 4 \%)$ and equal day and night cycle with free access to a standard pellet diet and water ad libitum. The study methodology was performed in accordance with international guidelines for Care and use of laboratory animals.

\section{Drugs and experimental design}

Drugs and chemicals

Cisplatin solution vial of Mylan, Greece $(1 \mathrm{mg} / \mathrm{ml})$ and Empagliflozin with the brand name (Jardiance) of Boehringer Ingelheim, Germany were purchased. Carboxymethyl cellulose (CMC) was obtained from Sigma Pharmaceutical Company, Quesna, Egypt. All other reagents were purchased from Sigma-Aldrich Co. (St. Louis, Missouri, USA) and were of analytical grade.

\section{Experimental design}

Rats were divided into 5 equal groups ( $n=8$ /group) and randomly allocated following completely randomized design using (RAND) function in EXCEL. Assessment of weight was done regularly.

Induction of acute kidney injury was done by intraperitoneal injection of a single dose (6 mg/ $\mathrm{kg}$ ) of Cisplatin-based on a previously published study of (Saad et al., 2001).

Empagliflozin was dissolved and suspended in 0.5\% CMC and administered orally by gavage needle in a dose of $10 \mathrm{mg} /$ kg/day (Lee et al., 2018).

Rats were grouped as follow

Control group: rats were fed $0.2 \mathrm{ml} 0.5 \% \mathrm{CMC}$ daily for 10 days.

Cisplatin group: rats were injected intraperitoneally by a single dose of Cisplatin (6 mg/kg).

Empagliflozin prophylactic group: 10 days before Cisplatin single dose, $10 \mathrm{mg} / \mathrm{kg}$ Empagliflozin was administered by oral gavage and on 10th day Cisplatin was injected as in Cisplatin group.

Empagliflozin therapeutic group: Cisplatin was injected as in Cisplatin group and $10 \mathrm{mg} / \mathrm{kg}$ Empagliflozin was administered by oral gavage for 10 days after Cisplatin single dose.

Empagliflozin group: Empagliflozin was administered in a dose of $10 \mathrm{mg} / \mathrm{kg}$ by oral gavage daily for 10 days.

\section{Animal sacrifice and sample collection}

At day 10 after Cisplatin administration, rats were euthanized via isoflurane inhalation anesthesia, blood samples were collected by cardiac puncture into vacuum tubes and serum was separated by centrifugation of blood at 4,000 rpm for $20 \mathrm{~min}$. Serum was kept in plastic Eppendorf at $-80{ }^{\circ} \mathrm{C}$ until further biochemical analysis.

Kidneys were extracted, weighed and washed with phosphate buffer. The left one was fixed in $10 \%$ neutral buffered formalin for histopathological examination and the right one was immediately stored at $-80{ }^{\circ} \mathrm{C}$ for later assay of nerve growth factor and oxidative stress parameters; malondialdehyde and reduced glutathione.

\section{Biochemical investigations}

Serum was used for assaying creatinine with diamond diagnostics kit (Diamond diagnostics company, Egypt) according to Jaffe colorimetric kinetic method (Vasiliades, 1976); Urea with the diamond diagnostic kit (Diamond diagnostics company, Egypt) according to Berthelot enzymatic colorimetric method (Fawcett and Scott, 1960). Uric acid was measured with uric acid liquicolor kit (Human diagnostics company, Germany) according to PAP (peroxidase-antiperoxidase) method (Fossati et al., 1980). Albumin was measured in serum with SPINREACT kit (SPINREACT, S.A./S.A.U., Spain) according to the bromocresol green colorimetric method (Rodkey, 1964) and glucose was assayed using SPINREACT kit (SPINREACT, S.A./S.A.U., Spain) according to GOD-POD (glucose oxidase and peroxidase) colorimetric method (Trinder, 1969).

\section{Determination of nerve growth factor beta (NGF- $\beta$ ) in kidney tissue}

Kidney was homogenized in $0.1 \mathrm{M}$ sodium phosphate buffer $\mathrm{pH} 7.4$ in a ratio of $1 \mathrm{~g}$ tissue to $4 \mathrm{ml}$ buffer and centrifuged at $4,000 \mathrm{rpm}$ for $20 \mathrm{~min}$ at $4{ }^{\circ} \mathrm{C}$, separated supernatant was stored at $-80{ }^{\circ} \mathrm{C}$ for further assay of tissue NGF- $\beta$ with commercially available rat NGF- $\beta$ ELISA kit from Chongqing Biospes Co., Ltd catalog number BEK1171 and results were expressed as ng/g tissue.

\section{Determination of malondialdehyde and reduced glutathione in kidney tissue}

Tissue levels of malondialdehyde and reduced glutathione were assayed by colorimetric methods according to Ohkawa et al. (1979) and Beutler et al. (1963) respectively with available kits produced by (Biodiagnostic company, Egypt).

\section{Histopathological evaluation}

Kidney tissues were fixed in $10 \%$ neutral buffered formalin and embedded in paraffin. $4 \mu \mathrm{m}$ thick sections were stained with hematoxylin and eosin (H\&E) and examined under a light microscope. A score of acute tubular necrosis (ATN) was done via evaluation of 10 fields of outer strip of medulla and cortex under 200× magnification of each slide and counting percentages of tubules showing cell necrosis, cast formation, loss of brush border and tubule dilation as previously reported by (Kim et al., 2015), this was graded as follows: $0=$ none, $1=$ $<10 \%, 2=11-25 \%, 3=26-45 \%, 4=46-75 \%, 5=>76 \%$.

\section{Immunohistochemical evaluation of caspase 3 expression and image analysis}

Briefly, $5 \mu \mathrm{m}$ sized paraffin-embedded tissue sections were de-paraffinized with xylene and hydrated in graded alcohol 
series, this was followed by thermal preparation in an acid medium. Then allowed to cool for 20 minutes, rinsed with distilled water and put in tris-buffered saline (TBS), endogenous peroxidase activity was quenched with $0.3 \% \mathrm{H}_{2} \mathrm{O}_{2}$ solution for 20 minutes, then sections were rinsed in TBS for 5 minutes 3 times and incubated with the primary antibody Rabbit polyclonal IgG to rat caspase 3 diluted 1 : 100 (Cusabio technology LCC, product code: CSB-PA786000) overnight at $4{ }^{\circ} \mathrm{C}$, then sequential adding of biotinylated secondary antibody against rabbit antibody for $1 \mathrm{~h}$ at $37^{\circ} \mathrm{C}$; streptavidin conjugated with horse-radish peroxidase (Streptavidin-HRP) (15 min incubation) and AEC substrate - HRP reaction dye (AEC substrate chromogen) with wash in between with TBS for 5 minutes 3 times, after that, slides were stained with DAB (3,39-diaminobenzidine tetrahydrochloride) (Sigma Chemical Co., St. Louis MO, USA), counterstained with hematoxylin and rinsed with distilled water. The sections were mounted and covered with coverslip and observed under a light microscope.

Calculation of percentage of cells showing positive immunostaining was done via Image $\mathrm{J}$ using IHC profiler plugin (Varghese et al., 2014) for 10 random fields from each slide.

\section{Statistical analysis}

Statistical analysis of results was performed using (Graph Pad Prism version 5 for Windows, 2007, Graph Pad software, Inc.). Data were expressed as mean \pm standard error of mean (SEM) and ATN score was expressed as median. Multiple comparisons were performed using One-way ANOVA followed by post hoc Tukey test, and Games Howell test for groups with unequal variances when assessed by Bartlett test while histopathology scoring was compared using Kruskal-Wallis test. The 0.05 level of probability was used as a criterion of significance.

\section{Results}

\section{Body weight change, absolute and relative kidney weight}

Initial body weight of rats showed no significant difference between groups. By comparing weight changes (difference) between initial and final body weight, it was found that Cisplatin group caused a significant decrease in body weight when compared to control group $(p<0.01)$. However, the Empagliflozin prophylactic group showed a non-significant decrease compared to Cisplatin group, while Empagliflozin therapeutic group showed a significant decrease compared to Cisplatin group $(p<0.001)$, both prophylactic and therapeutic groups showed a significant decrease compared to control group $(p<$ 0.001). Empagliflozin group showed no significant change compared to the control group (Fig. 1A, B). Absolute and relative kidney weight showed no significant change between different studied groups.
A

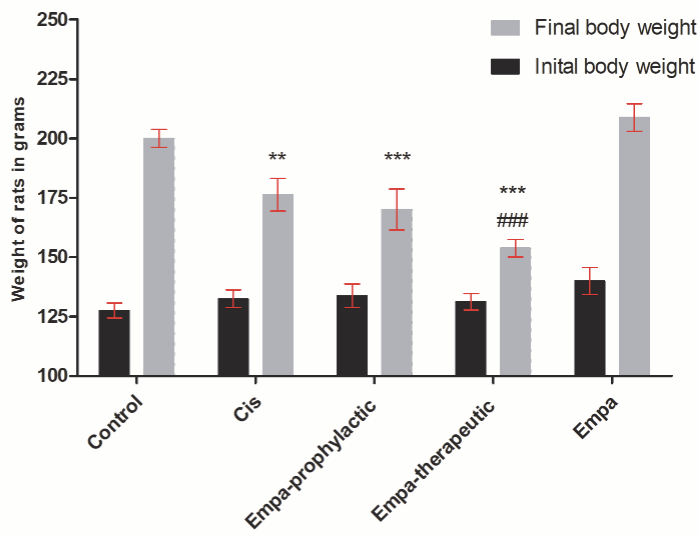

B

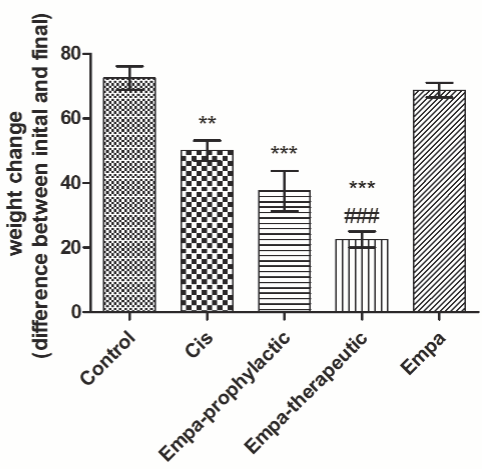

Fig. 1. Effect of Cisplatin and Empagliflozin on rat body weight changes.

A, initial and final body weight; B, weight change (the difference between initial and final body weight). Data are expressed as mean \pm SEM ( $n=8$ ); ${ }^{* *}=p<0.01 ;{ }^{* *}=p<0.001$ compared to control group, ${ }^{\# \#}=p<0.001$ compared to Cisplatin group. Cis = Cisplatin; Empa = Empagliflozin.

\section{Biochemical results}

Administration of Cisplatin caused significant increase in serum creatinine and urea $(p<001)$ compared to the control group, and Empagliflozin both prophylactic and therapeutic groups showed a significant decrease in serum creatinine and urea $(p<0.05,0<0.001)$ respectively in prophylactic group and $(p<0.001)$ in therapeutic group compared to Cisplatin group, while there was no significant difference between prophylactic and therapeutic Empagliflozin groups compared to each other.

Cisplatin and Empagliflozin did not affect serum uric acid and albumin as there were no significant changes between different studied groups except that Empagliflozin only group showed a significant decrease in uric acid compared to the control group.
Empagliflozin therapeutic group and Empagliflozin only group showed a significant increase in serum glucose level compared to the control group with no other significant changes in other groups (Table 1).

\section{Kidney tissue nerve growth factor-beta (NGF- $\beta$ ) results}

Administration of Cisplatin caused significant decrease in tissue levels of NGF- $\beta$ ( $p<0.01$ ) compared to the control group, but both prophylactic and therapeutic group of Empagliflozin did not show any significant difference either compared to Cisplatin or compared to each other, while showing significant decrease compared to the control group $(p<0.01)$ (Fig. 2). 
Table 1. Effect of Cisplatin and Empagliflozin on serum biochemical parameters

\begin{tabular}{lccccc}
\hline Group & Creatinine $(\mathrm{mg} / \mathrm{dl})$ & Urea $(\mathrm{mg} / \mathrm{dl})$ & Uric acid $(\mathrm{mg} / \mathrm{dl})$ & Albumin $(\mathrm{g} / \mathrm{dl})$ & Glucose $(\mathrm{mg} / \mathrm{dl})$ \\
\hline Control & $0.525 \pm 0.025$ & $49.25 \pm 2.491$ & $1.490 \pm 0.242$ & $3.813 \pm 0.079$ & $82.63 \pm 6.00$ \\
Cis & $0.850 \pm 0.057^{* * *}$ & $95.25 \pm 3.839^{* * *}$ & $1.518 \pm 0.233$ & $4.163 \pm 0.090$ & $100.4 \pm 6.936$ \\
Empa-prophylactic & $0.675 \pm 0.041^{\#}$ & $49.38 \pm 3.173^{\# \# \#}$ & $2.535 \pm 0.450$ & $3.863 \pm 0.122$ & $97.75 \pm 7.907$ \\
Empa-therapeutic & $0.637 \pm 0.032^{\# \#}$ & $50.13 \pm 4.809^{\# \# \#}$ & $3.194 \pm 0.559$ & $4.150 \pm 0.128$ & $111.1 \pm 4.486^{*}$ \\
Empa & $0.487 \pm 0.023$ & $39.63 \pm 1.647$ & $0.461 \pm 0.074^{*}$ & $3.913 \pm 0.130$ & $112.0 \pm 5.688^{*}$ \\
\hline
\end{tabular}

Values are expressed as mean \pm SEM

${ }^{*} p<0.05 ;{ }^{* *} p<0.01 ;{ }^{* * *} p<0.001$ compared to control group; ${ }^{\#} p<0.05, \# \# p<0.01, \# \#$ $p<0.001$ compared to Cisplatin group; Cis = Cisplatin; Empa $=$ Empagliflozin

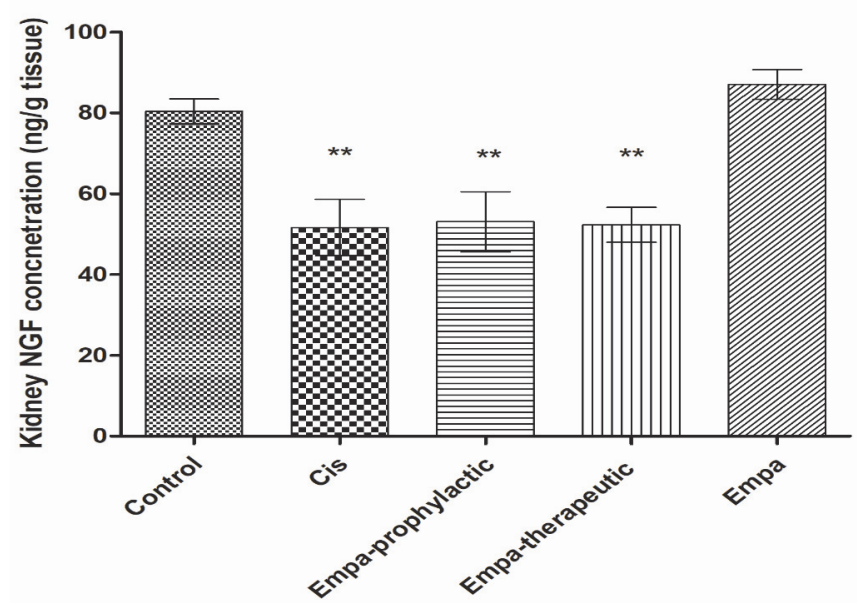

Fig. 2. Effect of Cisplatin and Empagliflozin on kidney tissue nerve growth factor- $\beta$.

Data are expressed as mean $\pm \operatorname{SEM}(n=8) ;^{*}=p<0.05 ;^{* *}=p<0.01$ compared to control group. Cis $=$ Cisplatin; Empa $=$ Empagliflozin.

\section{Kidney tissue malondialdehyde and reduced glutathione results}

Cisplatin injection caused a significant reduction in reduced glutathione with an elevation of malondialdehyde $(p<0.001)$ compared to control group, pretreatment with Empagliflozin resulted in significant elevation of reduced glutathione $(p<$ 0.001), while there was a non- significant change in the posttreatment group compared to Cisplatin group. Both pre and the posttreatment group did not show any significant difference in malondialdehyde level compared to the Cisplatin group (Fig. 3A, B).

\section{Histopathological results}

Administration of Cisplatin-induced acute tubular necrosis in the form of tubular degeneration, diffuse inflammatory infiltrate and severe interstitial hemorrhage with median ATN score (4.25) which was significant compared to control group $(p<0.001$ ) (Fig. 4A, B and Fig. 5). Empagliflozin prophylactic group showed significant decrease in the number of infiltrated cells, hemorrhage and tubular degeneration with median ATN score (1.25) compared to Cisplatin group $(p<0.05)$ (Fig. 4B, C
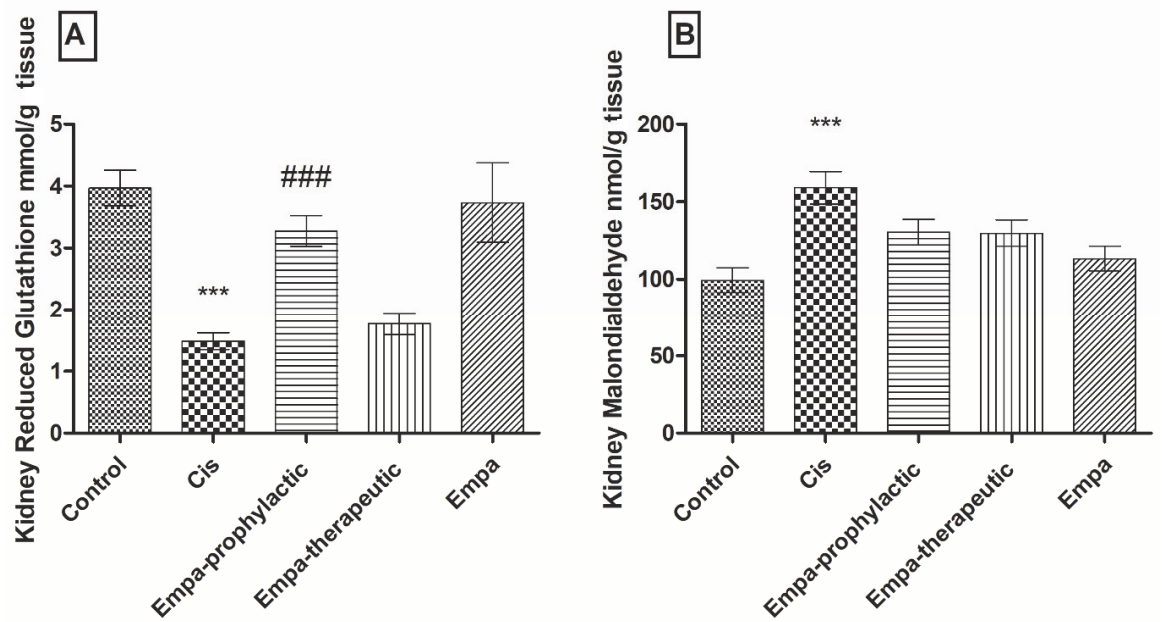

Fig. 3. Effect of Cisplatin and Empagliflozin on kidney tissue reduced glutathione $\mathbf{A}$ and malondialdehyde $\mathbf{B}$.

Data are expressed as mean $\pm \operatorname{SEM}(n=8) ;{ }^{* * *}=p<0.001$ compared to control group; $\# \# \#=p<0.001$ compared to Cisplatin group. Cis $=$ Cisplatin; Empa = Empagliflozin 

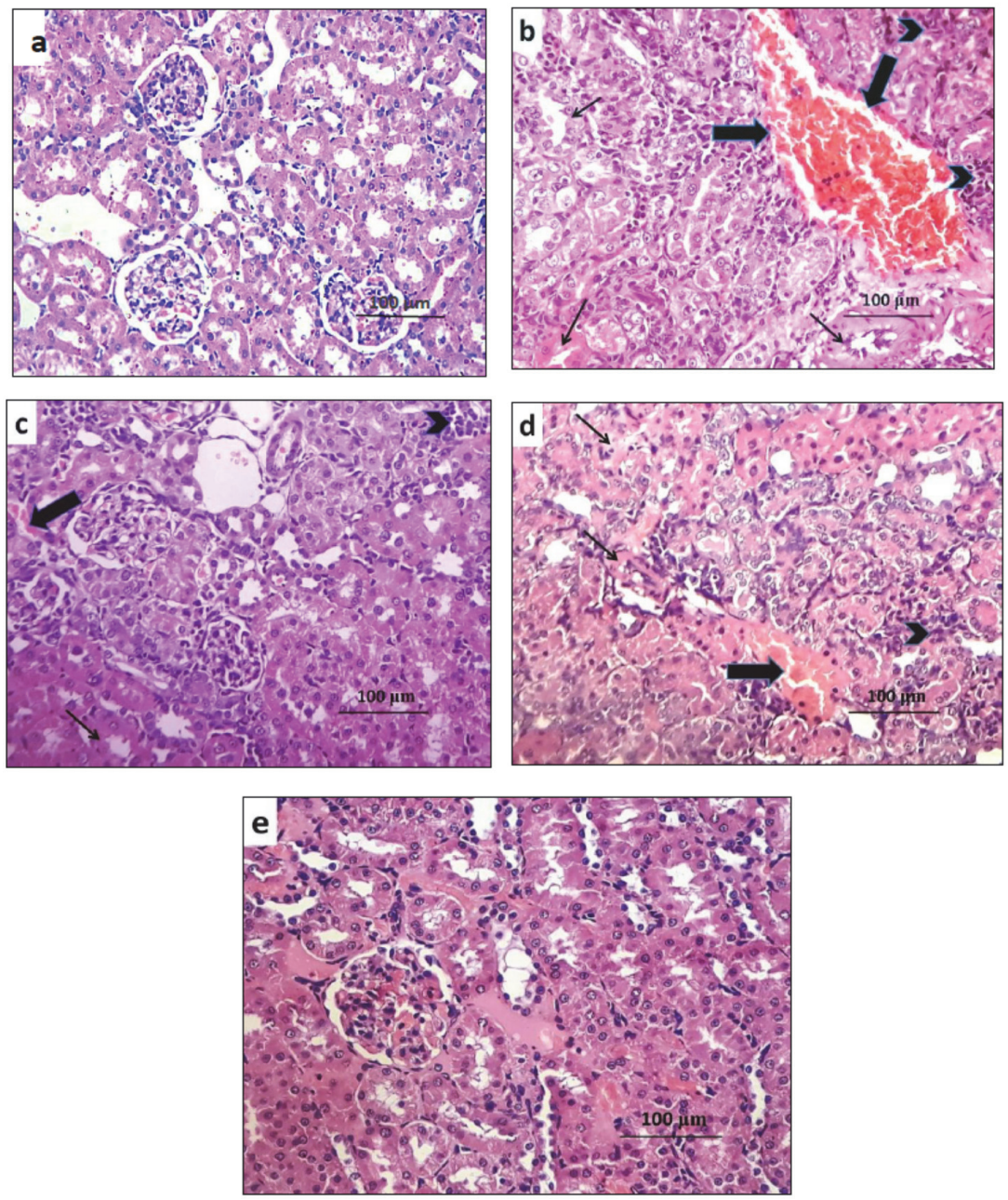

Fig. 4. Sections from the kidney (H\&E $\times 400)$ of $\mathbf{a}$, control group with normal appearance of the glomeruli, tubules, and interstitial tissue; $\mathbf{b}$, Cisplatin group showing massive tubular degeneration $(\boldsymbol{\uparrow})$, diffuse inflammatory cellular infiltration (Arrowhead) and severe interstitial hemorrhage ( $\mathbf{\uparrow})$; c, Cisplatin + Empagliflozin prophylactic group showing significant decrease in the number of infiltrated cells (Arrowhead), hemorrhage ( $\boldsymbol{\uparrow}$ ) and tubular degeneration $(\boldsymbol{\uparrow})$; d, Cisplatin + Empagliflozin treatment group showing mild inflammatory cellular infiltration (Arrowhead) with minimal hemorrhage $(\boldsymbol{\uparrow})$ and areas of tubular degeneration and necrosis ( $\boldsymbol{\uparrow})$; e, Empagliflozin group showing apparently normal glomeruli, tubules, and interstitium.

and Fig. 5), while Empagliflozin therapeutic group showed mild inflammatory infiltrate with minimal hemorrhage and areas of tubular degeneration and necrosis with median ATN score (3) which was not significantly different compared to Cisplatin group (Fig. 4B, D, Fig. 5). Empagliflozin group alone did not show a significant difference compared to the control group (Fig. 4A, E and Fig. 5).

\section{Immunohistochemical results of caspase 3}

Sections from Cisplatin group showed diffuse cytoplasmic and strong nuclear positive staining of caspase 3 compared to minimal staining of the control group (Fig. 6A, B). The use of Empagliflozin in the prophylactic group resulted in mild positive staining of caspase 3 compared to Cisplatin group (Fig. 6B, C), while Empagliflozin therapeutic group showed moderate positive staining of caspase 3 (Fig. 6B, D). Empagliflozin alone did not differ from the control group (Fig. 6A, E). Quantitative assay of percentage of positive cells showed that Cisplatin caused significant increase in percentage of positive cells compared to control group $(p<0.001)$, Empa-prophylactic group resulted in significant decrease in percentage of positive cells compared to Cisplatin group $(p<0.001)$, while Empa therapeutic group

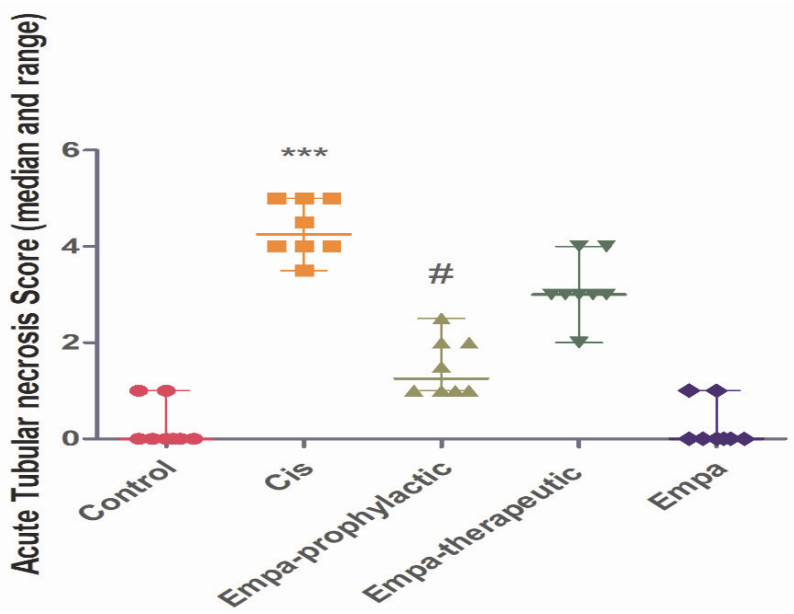

Fig. 5. Effect of Cisplatin and Empagliflozin on acute tubular necrosis factor score of histopathological sections.

Data are expressed as median and range $(n=8) ;{ }^{* * *}=p<0.001$ compared to control group; ${ }^{\#}=p<0.05$ compared to Cisplatin group. Cis = Cisplatin $;$ Empa $=$ Empagliflozin . 

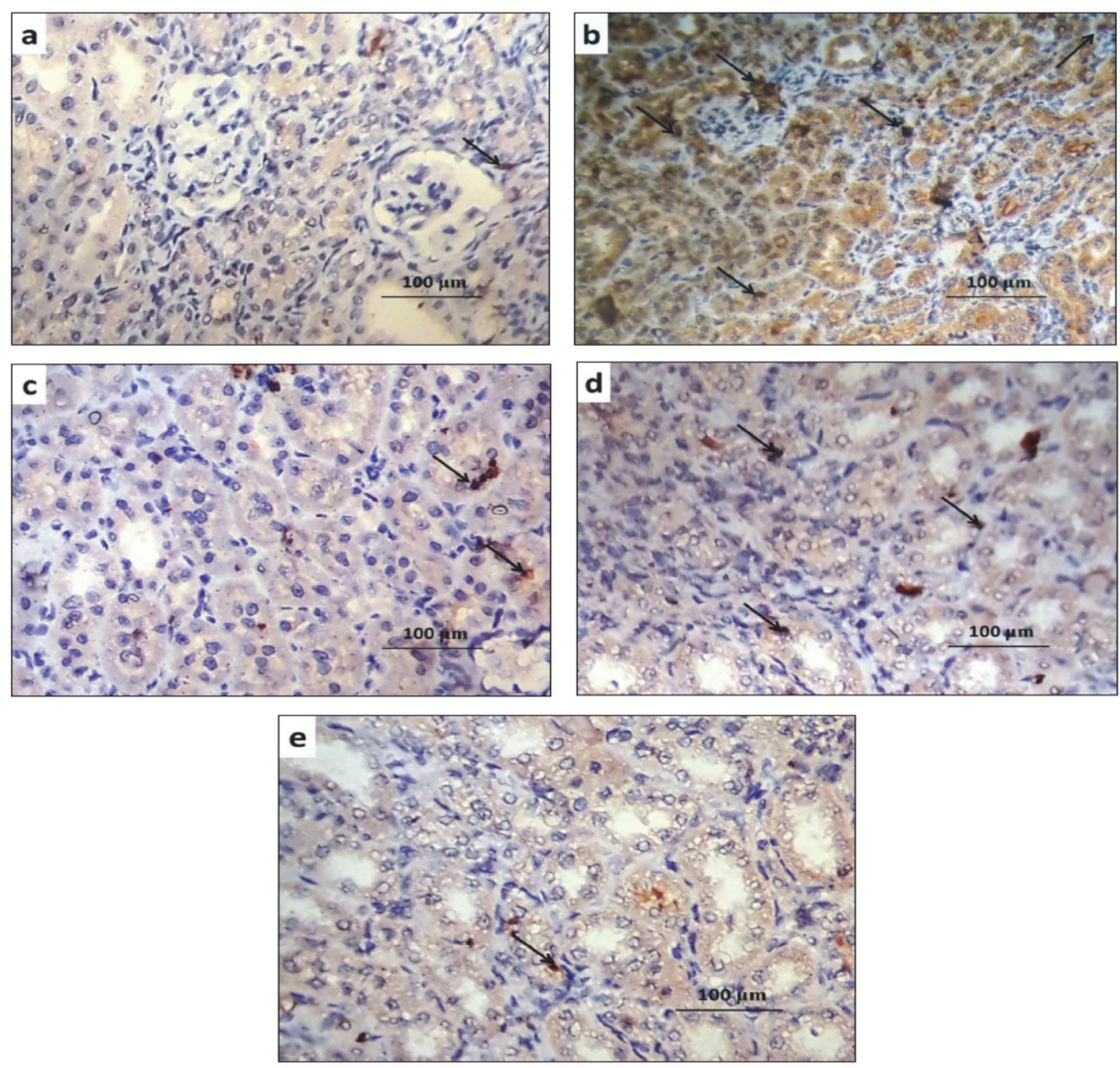

Fig. 6. Renal sections of immunohistochemical staining of caspase $3(\times 400)$ of a, control group showing minimal positive staining for caspase 3 ; b. Cisplatin group showing strong positive staining for caspase 3 ( $\uparrow$ ); $\mathbf{c}$, Cisplatin + Empagliflozin prophylactic group showing mild positive staining for caspase $3(\boldsymbol{\uparrow})$; d, Cisplatin + Empagliflozin treatment group revealing moderate positive staining for caspase 3 ( $\uparrow$ ); e, Empagliflozin group showing minimal positive staining for caspase 3 .

showed non-significant decrease when compared to Cisplatin group with ( $p$-value $=0.07)$. Empagliflozin group was non-significant compared to control group (Fig. 7).

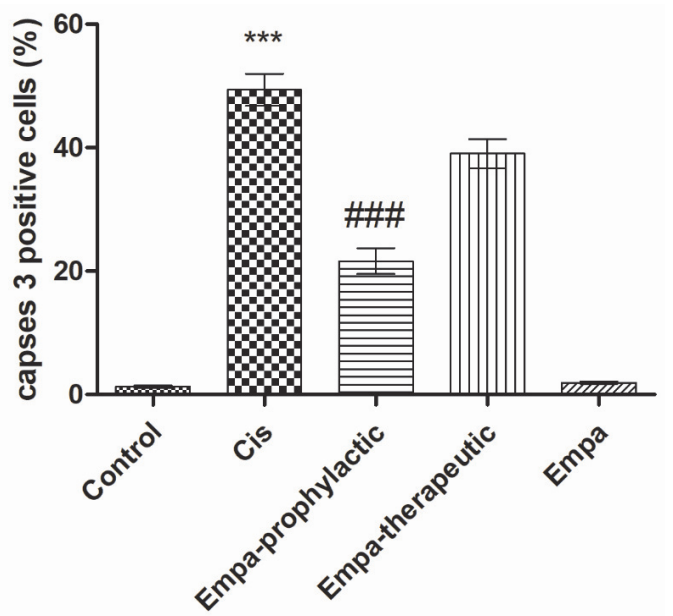

Fig. 7. Effect of Cisplatin and Empagliflozin on the percentage of caspase 3 positive cells.

Data are expressed as mean $\pm \operatorname{SEM}(n=8) ;{ }^{* * *}=p<0.001$ compared to control group; \#\#\# $=p<0.001$ compared to Cisplatin group. Cis $=$ Cisplatin $;$ Empa $=$ Empagliflozin

\section{Discussion}

Cisplatin is a powerful and reliable chemotherapeutic agent against many solid tumors. However, its use is limited by the development of nephrotoxicity which restricts high therapeutic doses. Therefore, finding an adjuvant therapy to ameliorate Cisplatin-induced nephrotoxicity is a priority. Strategies for preventing this injury nowadays include hydration and use of diuretics but unfortunately increased renal toxicity still occurs (Miller et al., 2010).

The purpose of this study was to investigate if SGLT2 inhibitor Empagliflozin, a drug used in treatment of type 2 diabetes, has a potential protective or therapeutic effect on acute kidney injury induced by Cisplatin and to elucidate its mechanism of action especially in relation to its effect on apoptosis marker caspase 3, oxidative stress and modulators of inflammation and cell differentiation via nerve growth factor assay. In addition, its effects on the other biochemical markers of kidney function and histopathology were assessed.

A single dose model of Cisplatin acute kidney toxicity was chosen in this study due to its clinical relevance as $30 \%$ of patients are exposed to acute kidney injury after a single dose, and due to its reproducibility as previously investigated (Ali et al., 2011; Humanes et al., 2012).

Cisplatin caused significant decrease in body weight of rats consistent with the results obtained by (Ali et al., 2011; Hu- 
manes et al., 2012) and may be attributed to decreased food intake due to nausea and anorexia induced by it, in addition to induction of polyurea and loss of nutrients in urine (Humanes et al., 2012; Liu et al., 2006).

In the present study, a single dose of Cisplatin caused a significant increase in serum creatinine and urea which is explained by the reduction of glomerular filtration rate because of acute tubular injury and necrosis. Accumulation of Cisplatin and conversion to active metabolite in renal tubular cells results in renal cell death, inflammation, fibro-genesis and tissue remodeling (Dugbartey et al., 2016; Taguchi et al., 2005). The main mechanism of this injury is oxidative stress as Cisplatin contains chloride ion which is hydrolyzed and releases hydroxyl radicles, but the use of antioxidant strategies had variable effects and decreased the anti-tumor effects of Cisplatin (Shirali and Perazella, 2014).

Although Cisplatin tends to elevate uric acid level due to deficient excretion as reported previously by (Silici et al., 2011), there was no statistically significant change in uric acid level between the different studies groups in the present study.

The nerve growth factor is a neurotrophic protein that maintains the critical balance between cell differentiation, survival, and death during development and adulthood. It is expressed not only in nervous tissue but also in many mammalian cells and acts on two types of receptors: tyrosine kinase receptor $\mathrm{A}(\mathrm{TrkA})$ and $\mathrm{p} 75$ neurotrophic receptor. The first one is related to cell differentiation, survival and thus protection of cells, while the latter is involved in induction of apoptosis and death of cells. It is also a strong inducer of epithelial cells and fibroblasts and has a strong relationship to inflammation and tissue repair (Micera et al., 2007). It was reported that it may be attributed to renal fibrosis through promoting epithelial-mesenchymal transition through TGF- $\beta$ signaling activation (Vizza et al., 2015).

It was demonstrated that NGF- $\beta$ signaling is important in human kidney and glomerular response to injury with the existence of NGF- $\beta$ and TrkA receptors in tubular and glomerular cells while p75 in interstitial and mesangial cells (Bonofiglio et al., 2007).

In the present work, Cisplatin caused a significant reduction of NGF- $\beta$ level in kidney tissue. This may demonstrate that there was a loss of its effect on cell survival as TrkA receptor exists in tubular cells, also, tubulointerstitial apoptosis and renal dysfunction was reported to be associated with upregulation of proapoptotic cytokines and such as TNF- $\alpha$ and TGF- $\beta$ and down-regulation of survival factor (Teteris et al., 2007) which was shown in this study; as reduction of NGF- $\beta$ was associated with increased caspase 3 immunostaining, the marker of apoptosis.

One of the main mechanisms of Cisplatin-induced acute kidney injury is oxidative stress via phospholipid damage, mitochondrial dysfunction and lysosomal hydrolase inhibition which leads to the accumulation reactive oxygen species. Activation of Cisplatin also leads to consumption of reduced glutathione which enhances the level of reactive oxygen species and cellular damage (Hosohata, 2016). Administration of Cisplatin in this study caused a reduction of reduced glutathione with an elevation of lipid peroxidation products.

It is now known that apoptosis or necrosis in tubular and tumor cells induced by Cisplatin is dose-dependent; as low dose may have relation to apoptosis while high dose leads to necrosis especially in renal tubules, and this necrosis may lead to activation of the inflammatory and immune responses. Another type of cell death called necroptosis or actively programmed necrosis may also occur in tubular cells as a result of an interaction of Cisplatin with different cellular organelles that have interacting pathways of necrosis and apoptosis (Sancho-Martínez et al., 2012).

Accumulation of Cisplatin inside proximal tubules and hence mitochondria which are abundant in this site, leads to mitochondrial dysfunction and reduction of ATP. DNA damage induced by Cisplatin causes activation of p53, increased reactive oxygen species and endoplasmic reticular stress, which all together lead to activation of caspase 9 and consequently caspases 3, 6, 7 that promote apoptosis and necrosis (Miller et al., 2010).

In the present study, there was mainly a histopathological picture of necrosis and degeneration of tubular cells in Cisplatin group. The level of caspase 3 has been also elevated, which is a marker of apoptotic pathway activation and is regarded as an irreversible event before cell death. This indicates the occurrence of necroptosis.

Empagliflozin is an inhibitor of SGLT2 transporter in kidney which showed a reno-protective effect in diabetic nephropathy with reduction of cardiovascular risks through control of hyperglycemia, lowering of blood pressure and body weight in addition to decreasing inflammation and glomerular hyperfiltration (Perrone-Filardi et al., 2017; Wanner, 2017). It was also shown that it may have a direct anti-inflammatory, antiapoptotic, anti-mitochondrial dysfunction and anti-oxidative effect, especially in studies of cardioprotection by these agents which seems to be independent of its effect on blood glucose level (Lahnwong et al., 2018).

In our work, administration of Empagliflozin orally in a dose of $10 \mathrm{mg} / \mathrm{kg}$ daily either prophylactic or therapeutic did not increase body weight compared to Cisplatin group, while when taken alone did not differ from control group (usual weight gain was preserved) and this is in contrary to studies that demonstrated decreased visceral adiposity by Empagliflozin (Kusaka et al., 2016; Xu et al., 2017). This may be explained by long-term administration of Empagliflozin in these studies in contrast to a short time in the current study. However, induction of hyperphagia and intake of fluids in response to glucosuria may have occurred in the group of Empagliflozin drug alone so weight gain was preserved.

Administration of Empagliflozin in both prophylactic and therapeutic groups resulted in a reduction of renal function parameters; serum creatinine and urea with no effect on albumin level. An increased serum glucose level was observed in Empagliflozin alone group which may be explained by compensatory hyperphagia (mentioned above). There was also a significant decrease in uric acid level in Empagliflozin only group compared to control, which may be attributed to increased uric acid excretion due to glucosuria (Chino et al., 2014).

Despite the importance of NGF- $\beta$ in cell survival, reduction of NGF- $\beta$ in Cisplatin group was also observed in both prophylactic and therapeutic Empagliflozin groups which indicates that the protective effect on the kidney is not through NGF- $\beta$.

Pretreatment with Empagliflozin caused improvement of the histopathological picture in form of decreased inflammatory infiltrate, tubule degeneration and hemorrhage and decreased ATN score. This was associated with elevation of reduced glutathione in kidney tissue. However, posttreatment with this drug showed a lower improvement than in the pretreatment group and a non-significant change of the ATN score compared to Cisplatin group. The improvement in pretreatment group may be related to antiapoptotic effect as demonstrated by mild positive immunohistochemical staining of caspase 3 in this group compared to moderate staining in 
posttreatment group, in addition to enhancing antioxidant defense in kidney tissue.

There are two types of SGLT family of glucose transporters expressed in kidney: SGLT 2 transporters are expressed in the S1 segment of proximal convoluted tubules and mediate $90 \%$ absorption of glucose, while SGLT1 transporters are present in S3 portion and account for reabsorption of the remaining glucose not absorbed by SGLT2 transporters (Szablewski, 2017). Some studies revealed that diabetic state may attenuate Cisplatin nephrotoxicity through upregulation of SGLT1 which may indicate a cytoprotective effect on the kidney (Debnam et al., 1995; Scott et al., 1989).

Empagliflozin inhibits SGLT2 transporters in kidney, which leads to increased activity of SGLT1 transporters as a compensatory mechanism (Rieg et al., 2014). This may have a cytoprotective effect as the SGLT1 transporters are involved in repair of plasma membrane integrity and tight junction as previously reported (Ikari et al., 2005a). Moreover, SGLT1 expressed in S3 partially suppressed peroxynitrite production hence protecting from Cisplatin nephrotoxicity (Ikari et al., 2005b). So, this may explain the renoprotective effect of Empagliflozin in this study although not investigated.

\section{Conclusions}

In conclusion, the present study demonstrated the protective effect of pretreatment with Empagliflozin on acute kidney injury induced by Cisplatin through reduction of apoptosis mainly and elevation of reduced glutathione, but not through nerve growth factor beta which was reduced in Cisplatin group. Further research is needed to investigate if Empagliflozin interferes with the antitumor effect of Cisplatin as most mechanisms of renal toxicity and tumor effects are interrelated.

\section{Conflict of interests}

The authors have no conflict of interests to declare.

\section{Authorship and funding}

This research did not receive any specific grant from funding agencies in the public, commercial, or not-for-profit sectors.

\section{References}

Ali BH, Abdelrahman AM, Al-Salam S, Sudhadevi M, AlMahruqi AS, Al-Husseni IS, et al. (2011). The effect of sildenafil on cisplatin nephrotoxicity in rats. Basic Clin Pharmacol Toxicol 109(4): 300-308. DOI: 10.1111/j.1742-7843.2011.00724.x.

Barabas K, Milner R, Lurie D, Adin C (2008). Cisplatin: a review of toxicities and therapeutic applications. Vet Comp Oncol 6(1): 1-18. DOI: 10.1111/j.1476-5829.2007.00142.x.

Beutler E, Duron O, Kelly BM (1963). Improved method for the determination of blood glutathione. J Lab Clin Med 61: 882-888.

Bonofiglio R, Antonucci MT, Papalia T, Romeo F, Capocasale G, Caroleo MC, et al. (2007). Nerve growth factor (NGF) and NGFreceptor expression in diseased human kidneys. J Nephrol 20(2) 186-195.

Chino Y, Samukawa Y, Sakai S, Nakai Y, Yamaguchi J, Nakanishi T, Tamai I (2014). SGLT2 inhibitor lowers serum uric acid through alteration of uric acid transport activity in renal tubule by increased glycosuria. Biopharm Drug Dispos 35(7): 391-404. DOI: 10.1002/bdd.1909.

Crona DJ, Faso A, Nishijima TF, McGraw KA, Galsky MD, Milowsky MI (2017). A systematic review of strategies to prevent cisplatin-induced nephrotoxicity. Oncologist 22(5): 609-619. DOI: 10.1634/theoncologist.2016-0319.
Dasari S, Tchounwou PB (2014). Cisplatin in cancer therapy: molecular mechanisms of action. Eur J Pharmacol 740: 364-378. DOI: 10.1016/j.ejphar.2014.07.025.

Debnam ES, Smith MW, Sharp PA, Srai SK, Turvey A, Keable SJ (1995). The effects of streptozotocin diabetes on sodium-glucose transporter (SGLT1) expression and function in rat jejunal and ileal villus-attached enterocytes. Pflugers Arch 430(2): 151-159.

Duffy EA, Fitzgerald W, Boyle K, Rohatgi R (2018). Nephrotoxicity: Evidence in patients receiving cisplatin therapy. Clin J Oncol Nurs 22(2): 175-183. DOI: 10.1188/18.CJON.175-183.

Dugbartey GJ, Peppone LJ, de Graaf IA (2016). An integrative view of cisplatin-induced renal and cardiac toxicities: Molecular mechanisms, current treatment challenges and potential protective measures. Toxicology 371: 58-66. DOI: 10.1016/j. tox.2016.10.001.

Fawcett JK, Scott JE (1960). A rapid and precise method for the determination of urea. J Clin Pathol 13(2): 156-159.

Fioretto P, Zambon A, Rossato M, Busetto L, Vettor R (2016). SGLT2 Inhibitors and the Diabetic Kidney. Diabetes Care 39(Suppl. 2): S165-S171. DOI: 10.2337/dcS15-3006.

Fossati P, Prencipe L, Berti G (1980). Use of 3,5-dichloro-2hydroxybenzenesulfonic acid/4-aminophenazone chromogenic system in direct enzymic assay of uric acid in serum and urine. Clin Chem 26(2): 227-231.

Hosohata K (2016). Role of oxidative stress in drug-induced kidney injury. Int J Mol Sci 17(11): 1826. DOI: 10.3390/ijms17111826.

Humanes B, Lazaro A, Camano S, Moreno-Gordaliza E, Lazaro JA, Blanco-Codesido M, et al. (2012). Cilastatin protects against cisplatin-induced nephrotoxicity without compromising its anticancer efficiency in rats. Kidney Int 82(6): 652-663. DOI: $10.1038 / \mathrm{ki} .2012 .199$

Ikari A, Nakano M, Suketa Y, Harada H, Takagi K (2005a). Reorganization of ZO-1 by sodium-dependent glucose transporter activation after heat stress in LLC-PK1 cells. J Cell Physiol 203(3): 471-478. DOI: 10.1002/jcp. 20234.

Ikari A, Nagatani Y, Tsukimoto M, Harada H, Miwa M, Takagi K (2005b). Sodium-dependent glucose transporter reduces peroxynitrite and cell injury caused by cisplatin in renal tubular epithelial cells. Biochim Biophys Acta 1717(2): 109-117. DOI: 10.1016/j.bbamem.2005.10.003.

Kim ES, Lee JS, Akram M, Kim KA, Shin YJ, Yu JH, Bae ON (2015). Protective activity of Dendropanax morbifera against cisplatininduced acute kidney injury. Kidney Blood Press Res 40(1): 1-12. DOI: $10.1159 / 000368466$.

Kusaka H, Koibuchi N, Hasegawa Y, Ogawa H, Kim-Mitsuyama S (2016). Empagliflozin lessened cardiac injury and reduced visceral adipocyte hypertrophy in prediabetic rats with metabolic syndrome. Cardiovasc Diabetol 15(1): 157. DOI: 10.1186/s12933016-0473-7.

Lahnwong S, Chattipakorn SC, Chattipakorn N (2018). Potential mechanisms responsible for cardioprotective effects of sodiumglucose co-transporter 2 inhibitors. Cardiovasc Diabetol 17(1): 101. DOI: $10.1186 / \mathrm{s} 12933-018-0745-5$.

Lee KA, Jin HY, Lee NY, Kim YJ, Park TS (2018). Effect of empagliflozin, a selective sodium-glucose cotransporter 2 inhibitor, on kidney and peripheral nerves in streptozotocininduced diabetic rats. Diabetes Metab J 42(4): DOI: 10.4093/ dmj.2017.0095.

Liu YL, Malik NM, Sanger GJ, Andrews PL (2006). Ghrelin alleviates cancer chemotherapy-associated dyspepsia in rodents. Cancer Chemother Pharmacol 58(3): 326-333. DOI: 10.1007/s00280005-0179-0.

Micera A, Lambiase A, Stampachiacchiere B, Bonini S, Bonini S, Levi-Schaffer F (2007). Nerve growth factor and tissue repair remodeling: trkA(NGFR) and p75(NTR), two receptors one fate. Cytokine Growth Factor Rev 18(3-4): 245-256. DOI: 10.1016/j. cytogfr.2007.04.004.

Miller RP, Tadagavadi RK, Ramesh G, Reeves WB (2010). Mechanisms of Cisplatin nephrotoxicity. Toxins 2(11): 24902518. DOI: 10.3390/toxins2112490.

Neumiller JJ (2014). Empagliflozin: a new sodium-glucose cotransporter 2 (SGLT2) inhibitor for the treatment of type 2 diabetes. Drugs Context 3: 212262. DOI: 10.7573/dic.212262. 
Ohkawa H, Ohishi N, Yagi K (1979). Assay for lipid peroxides in animal tissues by thiobarbituric acid reaction. Anal Biochem 95(2): 351-358. DOI: 10.1016/0003-2697(79)90738-3.

Peres LA, da Cunha AD, Jr. (2013). Acute nephrotoxicity of cisplatin: molecular mechanisms. J Bras Nefrol 35(4): 332-340. DOI: 10.5935/0101-2800.20130052.

Perrone-Filardi P, Avogaro A, Bonora E, Colivicchi F, Fioretto P, Maggioni AP, et al. (2017). Mechanisms linking empagliflozin to cardiovascular and renal protection. Int J Cardiol 241: 450-456. DOI: 10.1016/j.ijcard.2017.03.089.

Rieg T, Masuda T, Gerasimova M, Mayoux E, Platt K, Powell DR, et al. (2014). Increase in SGLT1-mediated transport explains renal glucose reabsorption during genetic and pharmacological SGLT2 inhibition in euglycemia. Am J Physiol Renal Physiol 306(2): F188-193. DOI: 10.1152/ajprenal.00518.2013.

Rodkey FL (1964). Binding of bromocresol green by human serum albumin. Arch Biochem Biophys 108(3): 510-513. DOI: 10.1016/0003-9861(64)90435-7.

Saad SY, Najjar TA, Noreddin AM, Al-Rikabi AC (2001). Effects of gemcitabine on cisplatin-induced nephrotoxicity in rats: scheduledependent study. Pharmacol Res 43(2): 193-198. DOI: 10.1006/ phrs.2000.0764.

Sancho-Martínez SM, Prieto-García L, Prieto M, López-Novoa JM, López-Hernández FJ (2012). Subcellular targets of cisplatin cytotoxicity: An integrated view. Pharmacol Ther 136(1): 35-55. DOI: 10.1016/j.pharmthera.2012.07.003.

Scott LA, Madan E, Valentovic MA (1989). Attenuation of cisplatin nephrotoxicity by streptozotocin-induced diabetes. Fundam Appl Toxicol 12(3): 530-539. DOI: 10.1016/0272-0590(89)90026-2.

Shirali AC, Perazella MA (2014). Tubulointerstitial injury associated with chemotherapeutic agents. Adv Chronic Kidney Dis 21(1): 56-63. DOI: 10.1053/j.ackd.2013.06.010.

Silici S, Ekmekcioglu O, Kanbur M, Deniz K (2011). The protective effect of royal jelly against cisplatin-induced renal oxidative stress in rats. World J Urol 29(1): 127-132. DOI: 10.1007/s00345-0100543-5.

Škrtić M, Cherney DZ (2015). Sodium-glucose cotransporter-2 inhibition and the potential for renal protection in diabetic nephropathy. Curr Opin Nephrol Hypertens 24(1): 96-103. DOI: 10.1097/MNH.0000000000000084.

Szablewski L (2017). Distribution of glucose transporters in renal diseases. J Biomed Sci 24(1): 64. DOI: 10.1186/s12929-0170371-7.

Taguchi T, Nazneen A, Abid MR, Razzaque MS (2005). Cisplatinassociated nephrotoxicity and pathological events. Contrib Nephrol 148: 107-121. DOI: 10.1159/000086055.

Teteris SA, Menahem SA, Perry G, Maguire JA, Dowling JP, Langham RG, et al. (2007). Dysregulated growth factor gene expression is associated with tubulointerstitial apoptosis and renal dysfunction. Kidney Int 71(10): 1044-1053. DOI: 10.1038/ sj.ki.5002176.

Trinder P (1969). Determination of blood glucose using an oxidaseperoxidase system with a non-carcinogenic chromogen. J Clin Pathol 22(2): 158-161.

Varghese F, Bukhari AB, Malhotra R, De A (2014). IHC Profiler: an open source plugin for the quantitative evaluation and automated scoring of immunohistochemistry images of human tissue samples. PLoS One 9(5): e96801. DOI: 10.1371/journal. pone.0096801.

Vasiliades J (1976). Reaction of alkaline sodium picrate with creatinine: I. Kinetics and mechanism of formation of the monocreatinine picric acid complex. Clin Chem 22(10): 1664-1671.

Vizza D, Perri A, Toteda G, Lupinacci S, Leone F, Gigliotti P, et al. (2015). Nerve growth factor exposure promotes tubular epithelial-mesenchymal transition via TGF-beta1 signaling activation. Growth Factors 33(3): 169-180. DOI: 10.3109/08977194.2015.1054989.

Wanner C (2017). EMPA-REG OUTCOME: The nephrologist's point of view. Am J Med 130(6S): S63-S72. DOI: 10.1016/j. amjmed.2017.04.007.

Xu L, Nagata N, Nagashimada M, Zhuge F, Ni Y, Chen G, et al. (2017). SGLT2 Inhibition by empagliflozin promotes fat utilization and browning and attenuates inflammation and insulin resistance by polarizing M2 macrophages in diet-induced obese mice. EBioMedicine 20: 137-149. DOI: 10.1016/j.ebiom.2017.05.028. 